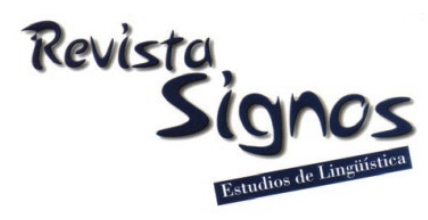

\title{
La sintaxis de los nombres de relación (no) simétrica*
}

\author{
The syntax of (non) symmetric relational nouns \\ María Mare \\ UNIVERSIDAD NACIONAL DEL COMAHUE/IPEHCS-CONICET \\ ARGENTINA \\ maria.mare@fahu.uncoma.edu.ar \\ Código ORCID: 0000-0002-9250-2467
}

Recibido: 16-VI-2018 / Aceptado: 07-XII-2018

DOI: $10.4067 /$ S0718-09342019000200457

\section{Resumen}

El presente trabajo tiene por objetivo desarrollar un análisis para los nombres de relación (no) simétrica en español que permita dar cuenta de sus propiedades semánticas, sintácticas y morfo-fonológicas. A tal fin, se revisan dos aspectos: por un lado, la relación entre el género y la noción de simetría ('Esteban es hermano de Ana' pero 'Ana es hermana/*hermano de Esteban') y, por el otro, las alternancias argumentales que se reconocen en estos nombres ('Esteban es hermano de Ana/Esteban y Ana son hermanos'). Lo novedoso de esta propuesta es que retoma algunas discusiones clásicas, como las referidas al género y a la predicación, y avanza en soluciones que permiten explicar un conjunto de nombres que constituyen una clase natural. Dado que el abordaje de este fenómeno se realiza desde un enfoque neoconstruccionista en el marco de la Morfología Distribuida, un aspecto central es la discusión sobre la individuación de raíces y la relación entre el componente sintáctico y el componente interpretativo. Finalmente, la propuesta busca extender al ámbito nominal la distinción entre elementos relacionales (categorizadores, PLACE) y no relacionales (Sintagmas Determinantes, raíces) para explorar cómo se establecen las relaciones de predicación y referencialidad.

Palabras Clave: Nombres (no) simétricos, raíces, categorizador nominal, estructura argumental. 


\begin{abstract}
The following paper aims to provide an analysis of (non) symmetric relational nouns in Spanish, accounting for their semantic, syntactic and morpho-phonological characteristics. Accordingly, two aspects are revisited: on the one hand, the relationship between gender and symmetry (Esteban es hermano de Ana 'Esteban is brother of Ana', but Ana es hermana/*hermano de Esteban 'Ana is sister/*brother of Esteban'); and, on the other hand, the alternations regarding the argument structure of these nouns (Esteban es bermano de Ana 'Esteban is brother of Ana'/Esteban y Ana son hermanos 'Esteban and Ana are brother and sister'). The novelty of this proposal is that it recovers classical discussions, such as the ones related to gender and predication, and sheds light on a group of nouns that conforms a natural class. Since this research is framed in a neoconstructionist approach like Distributed Morphology, it focuses on the discussion about root individuation and the relationship between syntactic derivation and the conceptual component. Last but not least, it extends the distinction between relational (categorizers, PLACE) and non-relational (DDPP and roots) elements into the nominal scope, in order to explore the difference in predication and referential expressions.
\end{abstract}

Key Words: (Non) symmetric nouns, roots, nominalizers, argument structure.

Pérez tenia un hermano. El hermano de Pérez. murió, pero el hermano de Pérez. nunca tuvo un hermano.

\title{
INTRODUCCIÓN
}

Los nombres de relación (no) simétrica como 'hermano', 'colega', 'amigo' y sus correspondientes en otras lenguas suponen una serie de desafíos para el análisis lingüístico. Desde el punto de vista semántico, diferentes propuestas se han centrado en el grado de simetría involucrado y en los factores que la determinan (Staroverov, 2007; Partee, 2008). Un factor que se ha considerado fundamental es la información de género, por lo que uno de los objetivos de la presente investigación es argumentar a favor de una propuesta que distinga las dimensiones semántica, morfosintáctica y morfofonológica de lo que se denomina 'género', de las cuáles únicamente la primera incide en la interpretación (no) simétrica.

Esta discusión es relevante para abordar las propiedades de las raíces en un modelo como la Morfología Distribuida, enfoque en el que se enmarca nuestra propuesta. El foco del debate es el criterio de identidad de las raíces, i.e., si la individuación de las raíces en la Sintaxis es fonológica (Borer, 2009), semántica o ninguna de los dos (Acquaviva, 2008). Por otro lado, y en estrecha relación con la individuación de raíces, hay otro punto de sumo interés: la manera en la que se determina la selección argumental, aspecto fundamental en la definición de 'relación simétrica' desde modelos neo-construccionistas. En este sentido, el punto de interés que presentan estos nombres tiene que ver con las alternancias de (1) y la agramaticalidad de (2), que indicarían algún tipo de codificación vinculada con la noción de pluralidad. 
(1) a. Bárbara y Andrea son hermanas.

b. Bárbara es hermana de Andrea.

(2) *Bárbara es hermana.

La pregunta que nos interesa explorar en relación a estos casos es si existe algún tipo de relación y en qué nivel estaría dada esa posible relación entre, por ejemplo (1a)

y (1b). Nuestro objetivo es demostrar de qué manera un abordaje neoconstruccionista en el que las raíces no están individuadas de ninguna manera resulta más explicativo que propuestas en las que esta información está codificada léxicamente.

El presente artículo se organiza de la siguiente manera. En el apartado 1, se desarrollan brevemente los supuestos generales del modelo teórico en el que se enmarca nuestro análisis. En el apartado 2 se discuten las propiedades de los nombres de relación simétrica en cuanto al género y a la relación entre género y número. La sección 3 se centra en la estructura argumental de estos nombres y la manera en la que se realizan los argumentos. Finalmente, presentamos las conclusiones de este recorrido.

\section{Morfología Distribuida. Supuestos generales}

El modelo teórico en el que se enmarca la discusión que presentamos aquí, la Morfología Distribuida (Halle \& Marantz, 1993), presenta una serie de supuestos que resultan centrales para la revisión de los nombres de relación (no) simétrica y ha habilitado el desarrollo de modelos de la estructura argumental inspirados en la propuesta de Hale y Kayser (1993), como Marantz (1997), Acedo-Matellán (2016). En este apartado, realizamos una descripción general de este modelo y nos centramos en las propuestas con respecto a la individuación de raíces y el rol de los categorizadores.

Los aspectos que caracterizan a la Morfología Distribuida (MD) son: (1) la Sintaxis como el único componente generativo (Syntax all-the-way-down), (2) la inserción tardía de los exponentes fonológicos y (3) la postulación de un componente postsintáctico denominado Estructura Morfológica (EM) en el que tienen lugar operaciones que pueden alterar el contexto para la inserción de vocabulario, pero que no tienen incidencia en el componente interpretativo. Así, el modelo de la gramática que propone la MD es el que representamos en (3), adaptado de Embick y Noyer (2005). 


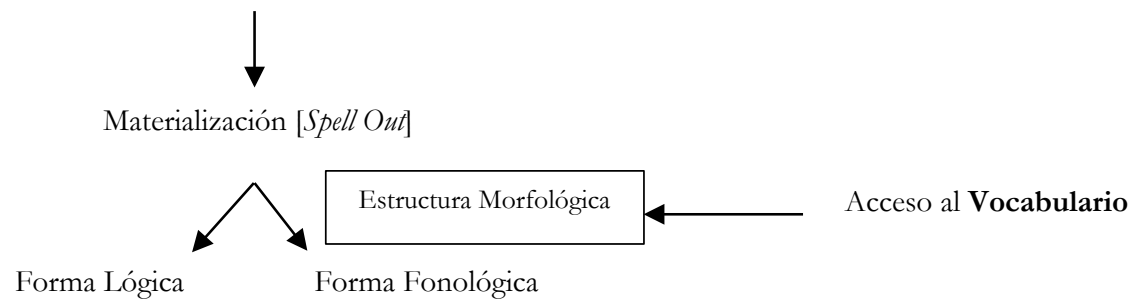

La primera característica marca el distanciamiento con las teorías lexicalistas que postulan un Lexicón en el que tienen lugar las operaciones de formación de palabras. Para la MD, la formación de palabras y de oraciones están sujetas a la misma operación: ensamble (externo e interno). Siguiendo este principio, se asume un único componente generativo en el que se forman estructuras que son enviadas de manera cíclica (fases) al componente interpretativo y al componente fonológico. Una de las operaciones de ensamble es la que se da entre una raíz y un categorizador, siguiendo el 'Supuesto de categorización' de Marantz $(1997,2001)$, cuyo antecedente es Chomsky (1970).

Supuesto de Categorización (Adaptado de Embick \& Marantz, 2008: 5) Las raíces no pueden aparecer (no pueden pronunciarse $o$ interpretarse) si no están categorizadas. Las raíces se categorizan a partir del ensamble sintáctico con núcleos funcionales que definen categorías.

Por ejemplo, la diferencia entre 'amar' y 'amor' tiene que ver con el tipo de categorizador con el que se ensambla la raíz $\sqrt{ }$ AM: verbal $(v)$ y nominal $(n)$, respectivamente. En el primer caso, tendremos una forma que luego se podrá ensamblar con las proyecciones propias del ámbito verbal en español (Tiempo, por ejemplo), mientras que en el segundo, los núcleos funcionales involucrados serán los propios del ámbito nominal (en principio, Número y Determinante). Entre las discusiones con respecto a los categorizadores tienen especial relevancia las referidas a los rasgos sintáctico-semánticos presentes en ellos y las que se vinculan con la manifestación de argumentos, es decir, si los categorizadores, además de categorizar, introducen argumentos (ver, por ejemplo, Harley \& Noyer, 2000). Estos aspectos son retomados en el próximo apartado, ya que resultan centrales para nuestro análisis sobre los nombres de relación simétrica.

La segunda característica, la inserción tardía, refiere a la concepción de la Gramática que asume la MD. La Gramática estaría compuesta por un conjunto de 
primitivos y un conjunto de reglas que derivan objetos complejos a partir de esos primitivos. Ese sistema se realiza en componentes de sonido y significado. La materialización en componentes de sonido supone la inserción tardía de exponentes fonológicos a morfemas abstractos, proceso que se denomina Inserción de Vocabulario. Los Ítems de Vocabulario son elementos listados en el Vocabulario (3), es decir, reglas que establecen las relaciones entre los rasgos fonológicos y el contenido de los morfemas abstractos.

(4) Formato de los ítems de vocabulario (IV)

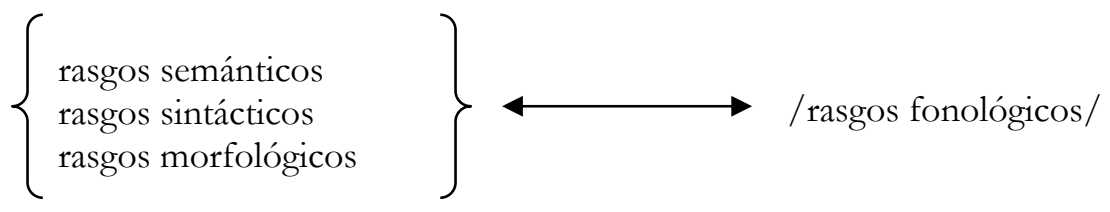

La Inserción de Vocabulario responde al Principio del Subconjunto (Halle, 1997), presentado a continuación.

Principio del Subconjunto [Subset Principle] (Halle, 1997: 128) (Traducción MM)

El exponente fonológico de un ítem de vocabulario se inserta en un morfema de una cadena terminal si el ítem es compatible con todos o un subconjunto de los rasgos especificados en el morfema terminal. La inserción no tiene lugar si el ítem de vocabulario contiene rasgos que no están presentes en el morfema. Cuando varios ítems satisfacen las condiciones para la inserción, debe ser elegido el ítem que es compatible con la mayor cantidad de rasgos especificados en el morfema terminal.

Un aspecto en discusión con respecto a la 'Inserción tardía' tiene que ver, nuevamente, con las raíces. De acuerdo con lo que desarrolla Embick (2015), en el caso por defecto, las raíces no estarían sujetas a inserción tardía, es decir, la mayoría de las raíces serían individuables a partir de sus rasgos fonológicos. Entre quienes asumen la inserción tardía también para las raíces, pueden reconocerse dos propuestas: (1) los IVs son licenciados por el contexto sintáctico inmediato, lo que pone en relieve el rol de los categorizadores (Harley \& Noyer, 2000) y (2) las raíces se comportan como cualquier otro IV y, por lo tanto, están sujetas a competencia a partir del Principio del Subconjunto (Siddiqi, 2009).

Finalmente, la postulación de un componente post-sintáctico denominado Estructura Morfológica o Morfología es uno de los ingredientes de la estructura de la Gramática que asume la MD. En este componente tendrían lugar operaciones que no afectan al componente interpretativo, sino que dan cuenta de la falta de isomorfismo entre la estructura que arroja la Sintaxis y la manera en la que esta estructura se materializa finalmente. En relación al tema que abordamos en este trabajo, es 
necesario mencionar que algunas líneas de la MD consideran que la concordancia es una operación que tiene lugar únicamente en este componente (Bobaljik, 2008), a partir del agregado de nodos disociados en los que se copia información presente en otros núcleos. En los próximos apartados retomaremos los aspectos mencionados aquí y profundizaremos en las discusiones planteadas en esta breve descripción del modelo.

\section{Sobre el género y el número de los nombres de relación (no) simétrica}

En el presente apartado nos centramos en las cuestiones referidas al género y al número de los nombres de relación (no) simétrica. Como anticipamos, la morfología de género parece incidir en la semántica del nombre y es lo que motiva que en la denominación utilicemos la negación entre paréntesis. Tal como define Partee (2008) una relación es simétrica ssi para todo $\mathrm{x}$, $\mathrm{y}$ : si $R(\mathrm{x}, \mathrm{y})$, entonces $R(\mathrm{y}, \mathrm{x})$. Esta implicación se cumple en los nombres relacionales que nos competen, cuando el género no está involucrado (por ejemplo, sibling ‘hermano' en inglés), pero no se satisface plenamente cuando hay información de género en el predicado en cuestión (por ejemplo, brother 'hermano'/sister 'hermana'). En estos casos, se considera que presentan una relación no-simétrica: una relación es no-simétrica ssi no es simétrica. Lo interesante de esta diferenciación es que la simetría o no-simetría de un predicado queda sujeta al dominio de la relación, i.e., si se trata de todos los seres humanos o si se trata de $\operatorname{humanos}_{[\mathrm{MACHO}]} /$ humanos $_{[\mathrm{HEMBRA}]}$. En este sentido, Partee (2008) señala que en el dominio de todas las personas, 'hermano de' (brother of) no es simétrico, ni asimétrico, sino no-simétrico: si x es 'hermano' de y, y puede ser 'hermano' de x o hermana' de x. Sin embargo, en el dominio de todos los humanos machos, 'hermano de' es simétrico. En la sección 2.1. revisamos algunas propuestas vinculadas con el rol del género y las dimensiones que propone Harris $(1991,1992)$ para dar cuenta del género en español.

En cuanto al número, hay al menos dos aspectos para señalar. Por un lado, estos nombres pueden aparecen en plural o en singular, pero siempre presuponen una relación simétrica, por lo que en el caso del singular, el segundo argumento debe recuperarse de alguna manera. Cuando los argumentos de estos nombres son plurales, establecen una relación recíproca no ambigua, algo que no sucede con otros predicados recíprocos: 'Esteban y Julio se aman', puede interpretarse de manera recíproca ('el uno al otro') o reflexiva ('a sí mismos’). Esa relación se mantiene en la materialización que llamaremos discontinua ( $\mathrm{X}$ es $\mathrm{N}_{\text {SIM }} d e \mathrm{Y}$ ). Si bien esta distribución de los argumentos será el centro de la discusión del apartado 3, el fenómeno mencionado nos lleva al segundo aspecto que nos compete aquí: la vinculación entre el número y el género. Como veremos, hay una serie de datos que indican que existe una estrecha relación entre estos dos tipos de rasgos, fundamentalmente, cuando las entidades involucradas en la predicación (no) simétrica presentan una denotación sexual opuesta (macho/hembra). En la sección 2.2. nos detenemos en esta discusión, 
para luego, en el apartado 2.3. presentar brevemente las condiciones que motivan la presencia de los morfemas nominales.

\subsection{El rol del género}

En diferentes trabajos sobre nombres relacionales se ha discutido la incidencia de la información de género a los fines interpretativos. Sin embargo, al momento de considerar que la información de género está presente en un objeto sintáctico que se caracteriza por ser nominal, se torna relevante la distinción entre raíces y categorizadores, que presentamos en el apartado anterior y también la autonomía de las dimensiones semántica, sintáctica y morfológica. Si, como argumentan diferentes autores (Saab, 2004; Percus, 2011; Estomba, 2017), la información de género se encuentra en el categorizador $n$, las raíces en sí mismas no ofrecerían distinciones en cuanto a la simetría. Esto significa que la interpretación no simétrica estaría dada recién cuando se ensambla el categorizador. Este aspecto es relevante, ya que en el marco de la teoría de las fases (Chomsky, 2001, 2008), algunas propuestas plantean que el complemento de los núcleos categorizadores es transferido a las interfaces cuando el SX está completamente construido (Marantz, 2001, 2007; Arad, 2003). Lo único que queda accesible luego de la transferencia es el núcleo X y el filo de la fase (el especificador del SX y los adjuntos). Desde esta perspectiva, la estructura relevante es la de (5).

(5) $\left[\ldots\left[n_{\text {GÉNERO/CLASE NOMINAL }}[\ldots \sqrt{ }\right.\right.$ RAÍZZ $\left.\left.]\right]\right]$

Una primera observación que surge de esto es la diferencia en términos de simetría entre $[\sqrt{ }$ RAÍZ $]$ y $\left[n_{\text {GÉN }}[\sqrt{ }\right.$ RAÍZZ $]$. Básicamente, podríamos decir que la Sintaxis opera con una raíz que podría considerarse simétrica, mientras que a la Forma Lógica llega un objeto sintáctico complejo que presenta, mínimamente, un categorizador $n$ para los casos que nos competen y que podría desencadenar una interpretación no simétrica. Como veremos en detalle en el apartado 3, la información del categorizador está condicionada por los rasgos de los argumentos que se ensamblan en torno a la $\sqrt{ }$ RAÍZ y que constituirían lo que Partee llama el dominio de la relación. Básicamente, la noción misma de simetría se obtiene de manera composicional y no como propiedad inherente de un nombre.

En este sentido, la discusión con respecto a la manera en la que se proyecta la información de género es central para esta parte de la discusión, por lo que consideraremos, en principio, dos conjuntos de datos: formas femeninas en singular (6) y contrastes entre masculino y femenino en plural y singular (7). Comencemos con el primer grupo.

(6) a. $* \mathrm{El} / \mathrm{La}$ pareja de Juan

b. El/La colega de Juan

c. ${ }^{*} \mathrm{El} / \mathrm{La}$ hermana de Juan 
Si bien tanto 'pareja', como 'colega' y 'hermana' presentan el exponente fonológico /a/, parece evidente que hay diferencias en cuanto a las condiciones que finalmente desencadenan la inserción de este exponente. En el caso de 'pareja', estamos ante un sustantivo epiceno (ver apartado 2.9.) en el que /a/ se inserta a partir de un rasgo $[+\mathrm{fem}]^{1}$ que nada tiene que ver con la denotación sexual de los participantes ('La pareja de Juan es Marcelo/Ana'; 'Juan y Ana/Marcelo son pareja'). 'Colega', en cambio, si bien presenta siempre este exponente, se diferencia de 'pareja' en tanto que no está especificado por un género en particular: no es un sustantivo femenino, aunque su terminación sea /a/, y tampoco es masculino. Sin embargo, la información relativa a la denotación sexual está presente en la estructura, ya que, el artículo y los modificadores con los que se combine presentan morfología de género en virtud de esa denotación. En este sentido, tiene el mismo comportamiento que los nombres con sufijo / ist-/ ('lingüista', 'deportista', 'dentista', etc.). Finalmente, en 'hermana' (6c), /a/ se relaciona con un rasgo [ $+\mathrm{fem}]$ motivado por la denotación sexual de los participantes y en este sentido, se opone a /o/ como [-fem] ('hermano'). En este último caso se aplicaría de manera clara la 'Regla del Clonaje Humano' (Harris, 1991), sobre la que volveremos inmediatamente.

Antes de avanzar con las propuestas sobre el género que consideramos aquí, queremos presentar el segundo grupo de datos (7).

(7) a. Un hermano es un amigo dado por la naturaleza. [J. B. Legouve]

b. Una hermana conoce todas tus historias.

c. Carlos y Ana son hermanos/*hermanas.

d. Ana y Estela son hermanas/*hermanos.

Los datos de (7) muestran diferencias relevantes con respecto a la denotación sexual de las formas en /o/ y en /a/. En el contraste entre (7a) y (7b), se reconoce una diferencia en cuanto a la denotación de las formas en singular: amplia en el caso de $(7 \mathrm{a})$, es decir, incluye referentes que no son únicamente [macho], siguiendo la terminología de Harris (1991); y estrecha en el caso de (7b), ya que todo lo que no se considere [hembra] (pace Harris, 1991) queda excluido de la denotación referencial. En las construcciones de (7c) y (7d), con referente explícito, se observa una distinción similar: 'hermanas' supone dos o más referentes [hembra], mientras que 'hermanos' no descarta la posibilidad de que uno de los referentes sea [hembra]. Para las formas en /o/ con denotación sexual amplia como (7a y 7c), Estomba (2017: 51 y siguientes) propone un análisis unificado basado en la hipótesis de hipermarcación de rasgos en la Sintaxis. Es decir, estos casos no serían nombres 'masculinos morfológicamente no marcados' (Harris, 1991), sino formas que presentan rasgos en oposición en la Sintaxis y, por lo tanto, están hipermarcados.

(8) Hipótesis de la hipermarcación de rasgos de género (Estomba, 2017: 51)

Un $\mathrm{N}$ humano con denotación sexual amplia está marcado [+fem] y [-fem]. 
Para avanzar en la discusión sobre los dos grupos que hemos definido aquí, es decir, los ejemplos (6) y (7), creemos relevante mencionar algunas distinciones que establece Harris (1991) sobre el género en español. En primer lugar, este autor argumenta a favor de la autonomía de las dimensiones semántica (sexo), sintáctica (género) y morfológica (marcador de palabra). Asimismo, demuestra que lo que denominamos masculino es el género por defecto, ya que es el que aparece incluso en ausencia de información de género, como sucede con la referencia a la preposición 'para' en el siguiente ejemplo: 'por ejemplo, mira: este [MASC] 'para' está de más (Harris, 1991: 43, ej. (20b)) (contra la hipótesis de hipermarcación de Estomba). Para reflejar este último aspecto, asume un rasgo privativo [fem], en lugar del sistema binario en el que ' + ' designa la forma marcada y '-' la forma no marcada (Harris, 1991).

A pesar de la necesidad de reconocer diferentes dimensiones y de destacar que la pertenencia a una declinación (dimensión morfológica) o a un género (dimensión sintáctica) no es totalmente predecible, Harris reconoce un conjunto de nombres con referencia humana en el que el género sí se predice de la información de sexo (macho/hembra), si bien existen algunas excepciones. Para dar cuenta de estos datos, propone la 'Regla del Clonaje Humano', mencionada previamente y presentada en detalle a continuación. Cabe aclarar que el rasgo [humano] propuesto por Harris (1991), se entiende en un sentido amplio para predecir la existencia de pares machohembra a partir de una misma base léxica. Esto significa que en el par 'león/leona' se reconoce también el rasgo [humano], que no estaría presente en 'jirafa', por ejemplo.

(9) Regla de clonaje humano (1991: 51) (Traducción MM).

Para cada entrada léxica $L$ de una base nominal que contenga la especificación semántica 'humano' sin especificación de sexo, Clonaje Humano reemplaza L por un par de entradas $\mathrm{L}_{\mathrm{m}[\text { asculino] }}$ y $\mathrm{L}_{\mathrm{f}[\mathrm{emenino} \text {, }}$ cada una de ellas idéntica a $\mathrm{L}$ excepto por la adición de la especificación semántica 'macho’ y ‘hembra', respectivamente.

(10) a. Clonaje humano

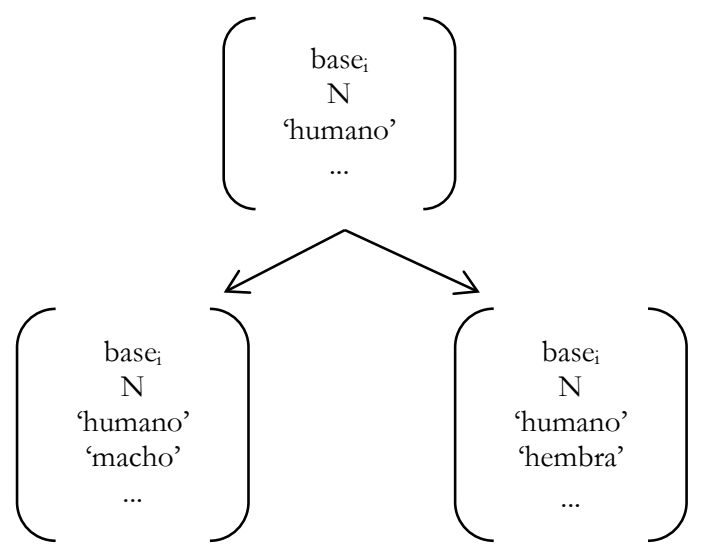




\section{b. Género humano}

'Hembra' $\rightarrow$ femenino / [ _ _ 'humano']

c. Regla de marcado del femenino

Femenino $\rightarrow$ ]a

[Adaptado de Harris, 1991]

Si revisamos los datos de (6) a la luz de esta propuesta, vemos que 'pareja' no está sujeta a 'clonaje humano', ya que desde ningún punto de vista podría sostenerse la presencia del rasgo [humano]. Es decir, el femenino de 'pareja' no es predecible y sería equivalente al de 'mesa'. Por su parte, el par 'hermano/hermana' responde perfectamente a los postulados de (9) y (10), por lo que 'hermana' es el resultado de la aplicación de la 'Regla de clonaje humano' sobre 'herman-' [N, 'humano', ...] (10a), que para el rasgo [hembra] desencadena la inserción del rasgo [femenino] (10b), que se materializa como /a/ (10c). Queda ahora por determinar el caso de 'colega'. De acuerdo con Harris, este nombre también está sujeto a la 'Regla de clonaje humano', si bien no hay una manifestación morfológica en el propio nombre, ya que se reconoce morfología distintiva asociable a la denotación sexual en el determinante y otros posibles modificadores ('el colega simpático'/'la colega simpática'). Harris plantea que la /a/ en este caso es un marcador de clase ya especificado en la base léxica (un diacrítico). En este ejemplo en particular, se observa de manera clara la distinción entre la dimensión semántica ('colega'> humano $>$ macho/hembra), sintáctica (femenino/masculino) y morfológica (coleg- ]a).

Es evidente que para los casos que estamos discutiendo 'colega' constituye una excepción que Harris resuelve en la entrada léxica y nosotros no resolveremos de manera mucho más elegante, aunque sí, desde una propuesta coherente con los postulados que asumimos en este trabajo. Sin embargo, más allá del carácter no predecible del morfema /a/ en 'colega', para un modelo de inserción tardía como la Morfología Distribuida, en el que los ítems de vocabulario compiten por la inserción en un nodo, este hecho no es en absoluto trivial. Básicamente, cabe preguntarse qué rasgos motivan la inserción de /a/, si descartamos la hipótesis de que hay más de un morfema nominal /a/ en el Vocabulario del español. Asimismo, en un modelo de inserción tardía (o incluso en una propuesta más lexicista como la de Harris, 1991), no parece deseable que la interpretación semántica se defina a partir de un diacrítico que simplemente indica la pertenencia a una clase (dimensión morfológica o incluso morfofonológica).

\subsection{El rol del número en relación al género}

Para avanzar en la propuesta sobre el género de los nombres de relación simétrica, resulta central volver sobre el conjunto de (7) y particularmente sobre la propuesta de Estomba, que a diferencia de Harris, vincula la información de género con la proyección de Número. De acuerdo con Estomba (2017: 44), “el género sintáctico 
constituye la gramaticalización del carácter individuante de la categoría nominal, más allá de si se trata de un N humano o no humano". Dado que, de acuerdo con Borer (2005) es la proyección Núm con el rasgo [div(isión)] la que determina la individuación de los nombres, Estomba (2017) propone que cuando $n$ se ensambla con Núm [div] marca con un rasgo de género al núcleo de su complemento, que luego se especificará como [+fem] o [-fem] en la Sintaxis o en la Morfología. Como puede seguirse de esta exposición, la ausencia de género en el sentido de Harris es un problema para la individuación, de ahí la necesidad de postular rasgos binarios y no privativos. Es decir, más allá de cuáles sean los rasgos sintáctico-semánticos para el exponente fonológico /o/, los rasgos de género deben estar presentes en $n$ a los fines de la individuación. De acuerdo con Estomba, para los nombres con el rasgo [humano], [género] queda especificado como [+fem] o [-fem] en la Sintaxis, de acuerdo con la Regla de Clonaje Humano, mientras que en los nombres no humanos esta asignación tiene lugar en la Morfología a partir de la siguiente condición, que sigue la propuesta de Saab (2004, 2008).

\section{(11) Condición de exhaustividad de género}

Asígnese [+fem] o [-fem] en la Morfología a todo $n$ que no haya recibido dichos rasgos en la Sintaxis.

Ahora bien, como adelantamos en el subapartado anterior, la denotación sexual amplia del masculino es entendida por Estomba como un caso de hipermarcación de rasgos. Así, la arquitectura de rasgos de género subyacente a (12a) y (12b) sería la misma, mientras que la diferencia radicaría en el rasgo de Número.

(12) a. Un hermano es un amigo dado por la naturaleza.

b. Ana y Esteban son los hermanos de Carla.

Si bien esta propuesta resulta sumamente interesante, pierde una generalización que alcanza Halle (1997) y que desarrolla Saab (2008) en cuanto a la hipermarcación de rasgos y sus consecuencias para los rasgos de número: la hipermarcación motiva la presencia del rasgo [+PL] en Número. Ahora bien, ¿cómo se puede dar cuenta de la denotación sexual amplia del masculino singular? Cabe mencionar que esta interpretación se asocia o bien a la interpretación genérica del Sintagma Determinante (SD), o bien a la falta de información sobre la denotación sexual, algo que también puede suceder con formas de plural ('Los hermanos son los amigos dados por la naturaleza' ${ }^{2}$. Sin embargo, la lectura no genérica de grupo heterogéneo en cuanto a la denotación sexual no admite formas del singular: 'Los hermanos de Carla' (denotación sexual amplia) vs. 'Un/El hermano de Carla' (denotación sexual estrecha).

Para dar cuenta de esta diferencia y mantener la relación entre la hipermarcación y la pluralidad, consideramos una cuarta opción para el género: aquella en la que no esté especificado el valor del rasgo [ $\alpha \mathrm{fem}]$. Considerar esta opción nos permite captar la 
propuesta de Estomba en cuanto a la relevancia del género a los fines de la individuación, pero también los casos que llevan a Harris (1991) a proponer que el masculino es la ausencia de género. En este sentido, el ensamble de Núm [div] marca con un rasgo [ $\alpha \mathrm{fem}]$ al $n$ que se especificará como [+fem], [-fem] o no se especificará, quedando como $[\alpha \mathrm{fem}]$ y habilitando la lectura genérica. Finalmente, si se especifica como [+fem, -fem], es decir, si hay hipermarcación, Núm obligatoriamente presenta el rasgo $[+\mathrm{PL}]$.

Para los nombres humanos, se ha asumido que la información de género es relevante para el componente interpretativo, independientemente de cómo se materialice, es decir, de cuál sea el exponente fonológico que se inserta finalmente. En este sentido se justifica que la información de género esté presente en la Sintaxis (ver Estomba, 2017). Sin embargo, consideramos que la única información pertinente a los fines interpretativos es la que refiere a la denotación sexual [macho]/[hembra], independientemente de cómo se especifique su género. De hecho, este es el punto de partida de nuestra discusión con respecto a la simetría o no simetría de los nombres que nos competen: tal como se mencionó previamente, la evaluación en estos términos se seguiría del dominio de la relación, dominio que se establece en virtud de la referencia a los participantes involucrados. Por esto proponemos que el único rasgo de género que aparece en la Sintaxis es [ $\alpha \mathrm{fem}]$ y es un rasgo pertinente a los fines de la individuación (siguiendo a Estomba) y que funcionará como instructivo para la concordancia, cuando se especifique en el componente Morfológico. Una aclaración relevante para la discusión en el próximo apartado es que el carácter individuante que diferencia la categoría nominal de otras categorías requiere de la presencia de $n$ y del rasgo $[\alpha \mathrm{fem}]$. La ausencia de $n \mathrm{y}[\alpha \mathrm{fem}]$ no permite obtener la referencia individuada (siguiendo a Estomba, con las adaptaciones correspondientes).

Antes de especificar los aspectos relativos a los marcadores de palabra y la información de género, nos interesa revisar los casos de denotación sexual amplia ('Los hermanos de Ana') y de interpretación genérica ('Un hermano es un amigo...'/'Los hermanos son los amigos...'). Para el primer caso, la raíz se ensambla con un $n$ con el rasgo [humano] y en este caso, la regla del clonaje humano se aplica permitiendo la convivencia de las dos especificaciones semánticas, i.e. [macho] y [hembra]. En la Morfología el rasgo [ $\alpha$ fem] se especificará en relación a estos rasgos opuestos, lo que produce la hipermarcación [+fem] para [hembra] y [-fem] para [macho]. La hipermarcación tendrá consecuencias en la concordancia, mientras que la doble marcación semántica desencadenará que Núm sea [+PL]. En el segundo caso, proponemos que el ensamble se da con un categorizador nominal que carece del rasgo [humano] y, por lo tanto, la 'Regla del clonaje humano' no se aplica. Así, la especificación de $[\alpha \mathrm{fem}]$ no se da en relación a los rasgos [macho]/[hembra], ya que están ausentes y, dado que esa raíz no se vincula prototípicamente a un rasgo de género particular, queda marcada como $[\alpha f e m] .{ }^{3}$ 
Las estructuras que asumimos, entonces, se presentan a continuación.

(13) a. Esquema para 'hermana' ('hermano/hermana')

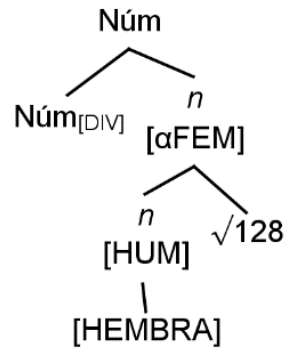

b. Especificación en la Morfología: $n_{[\mathrm{Hembra}] /[\alpha \mathrm{fem}]} \rightarrow n_{[+\mathrm{fem}]}$

(14) Esquema para ‘hermano’ (genérico)

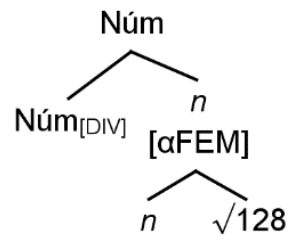

(15) Esquema para 'hermanos’ (macho/hembra)

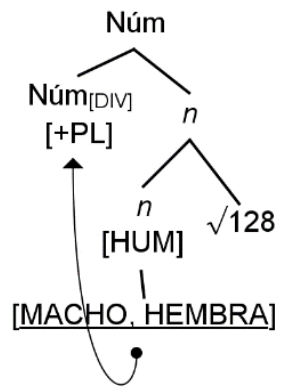

\subsection{Los marcadores de palabra}

Ahora bien, como señalan Harris (1992) y Kihm (2005), no hay una correlación entre marcadores de palabras e información de género. Si volvemos al nombre 'colega', encontramos que puede formar parte del siguiente sintagma: 'un colega famoso'. Hay algo en esa construcción que bloquea la presencia de 'una' y de 'famosa', independientemente del morfema '-a' de 'colega'. No solo eso, sino que no hay coincidencia entre estas palaras en su marcación ('un- $\varnothing$ ', 'coleg-a' y 'famos-o'), pero aun así se reconoce una relación entre ellas que definimos como 'concordancia'. Por este tipo de evidencia empírica, distinguimos la información de género $[\alpha$ fem], como condición de individuación de los nombres y relevante a los fines de la concordancia, 
de la información de clase/marcador de palabra, como condición de buena formación de palabras, vinculada a aspectos de la interfaz morfología-fonología. En el caso de los nombres humanos, sujetos a la 'Regla del clonaje humano', la información de género [+fem] (vinculada a [hembra]) desencadena la inserción del marcador de palabra correspondiente a la Clase II de Harris (1992), i.e., /a/, a menos que exista una especificación morfofonológica independiente para la cadena fonológica en cuestión.

Seguimos, entonces, la propuesta de Harris (1991, 1992) de que en el dominio morfofonológico los nombres del español pertenecen a diferentes clases, a partir de las cuales, se determina el marcador de palabras que se inserta. Así, por ejemplo, a la Clase I corresponde el exponente fonológico /o/, a la Clase II el exponente fonológico /a/ y a la Clase III un exponente fonológico /Ø/, que según sea la silabificación, dará lugar a la inserción de una vocal epentética /e/.

En el caso prototípico, los sustantivos del español pertenecen a las Clases I y II. Con respecto a la Clase II, a excepción de un número reducido de nombres, hay una correlación entre [+fem] y Clase II (/a/), algo que Harris recupera como una regla de redundancia. Así, si volvemos sobre la terna que inicia nuestra exposición ('pareja', 'hermana', 'colega'), podemos decir que una vez en el componente morfológico, 'pareja' y 'hermana' especifican su rasgo [ $\alpha \mathrm{fem}]$ como $[+\mathrm{fem}]$, lo que resulta, luego, en la marcación esperable como Clase II. 'Colega', en cambio, puede especificar su rasgo como [-fem] o bien registrar hipermarcación [+fem, -fem]. Sin embargo, ninguna de

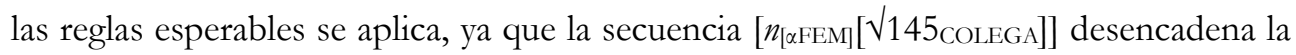
marcación como Clase II.

\subsection{Resumen}

A lo largo de este apartado discutimos algunos aspectos que consideramos centrales con respecto al género y al número en los nombres de relación simétrica. Con Estomba (2017) asumimos que el género es la gramaticalización del carácter individuante de los nombres y que está presente en el categorizador nominal, sin embargo, nos diferenciamos de su propuesta dado que consideramos que el rasgo adquirido por un $n$ seleccionado por $\mathrm{Núm}_{[\mathrm{DIV}]}$ es $[\alpha \mathrm{fem}]$, i.e., un rasgo con valores no especificados. Para todos los casos, estos valores se especificarán recién en componente morfológico a los fines de la concordancia. Asimismo, siguiendo la 'Regla del clonaje humano', asumimos que los nombres humanos, presenta rasgos de denotación sexual ([macho]/[hembra], siguiendo a Harris, 1991). También acordamos con la propuesta de la hipermarcación de rasgos, para aquellos casos en los que la denotación sexual incluye entidades [macho] y [hembra]. Es decir, estos nombres estarán marcados en la Morfología como [+fem, -fem]. A diferencia de Estomba, planteamos que la hipermarcación resulta necesariamente en la especificación de número [+PL]. Así, nuestro sistema de rasgos presenta [+fem], [-fem], [ $\alpha$ fem] y [+fem, -fem], los tres primeros pueden combinarse con Núm ${ }_{[+/-P L}$, mientras que el 
último bloquea la presencia del rasgo [-PL]. El rasgo [ $\alpha \mathrm{fem}]$ es la marcación por defecto que, en el caso de los nombres humanos, desencadena la lectura genérica (en combinación con otros rasgos). Finalmente, distinguimos entre género, por un lado, y marcador de palabra, por el otro. El género es relevante a los fines de la individuación (Sintaxis) y de la concordancia (Morfología), pero no se corresponde necesariamente con los marcadores de palabra.

Volviendo al problema inicial con respecto a la relación entre la interpretación simétrica y el género, la delimitación realizada nos permite centrar la discusión en el dominio estrictamente sintáctico-semántico, dejando al margen los aspectos morfofonológicos. Así, la interpretación simétrica estará dada en tanto exista una relación en la que los referentes presenten denotación sexual idéntica (dominio de alcance estrecho). De lo contrario, la interpretación será no simétrica, ya que el objeto sintáctico enviado al componente interpretativo tiene rasgos que determinan un dominio de alcance amplio ([macho]/[hembra]), pero el tipo de predicación interpretable para esa raíz es simétrica: si $R(x, y)$, entonces $R(y, x)$, donde $R$ es $\sqrt{ } 143$ (no $\left.\left[n_{[\text {HUM...] }}[\sqrt{ } 143]\right]\right)$.

\section{La estructura argumental de los nombres de relación (no)simétrica}

Como mencionamos en la introducción, los nombres de relación (no) simétrica suponen un desafío también para los abordajes con respecto a su estructura argumental, ya que manifiestan la alternancia que denominamos continua/discontinua ('Esteban y Miguel son hermanos'/'Esteban es hermano de Miguel'). Independientemente de cómo se proyecten los argumentos, la interpretación simétrica se mantiene, como así también las interpretaciones condicionadas por la denotación sexual de los referentes. Asimismo, estos nombres pueden realizarse como predicados ('Ellos son hermanos') o como argumentos ('los hermanos'), diferencia en la que también nos detendremos aquí.

\subsection{Los nombres de relación (no) simétrica en un modelo neo- construccionista}

En la propuesta que adoptamos, las diferencias en la distribución de las raíces se siguen de su interacción con rasgos sintáctico-semánticos en la estructura. En los modelos neo-construccionistas, la combinación de raíces con elementos relacionales (Mateu, 2002), como n, v, Path o Place, da lugar a distinciones de significado y de selección argumental. Es decir, las raíces no proyectan esta información, sino que la estructura que se forma en la Sintaxis es interpretada de una u otra manera en el componente interpretativo (ver Borer, 2005 y el recorrido en Demonte, 2015). En esta línea y siguiendo la propuesta de Acedo-Matellán (2016) asumimos que las raíces presentan el mismo comportamiento que los SSDD: no proyectan estructura y se 
interpretan de acuerdo a la posición que ocupan en la configuración sintáctica (ver también Marantz, 2013). Además, se relacionan con núcleos funcionales como $n \mathrm{y}$ Place, en los casos que analizamos aquí. Finalmente, una estructura no puede proyectar un especificador si no tiene un complemento. Esto significa que los predicados inergativos son subyacentemente predicados transitivos. Con todo, la interpretación semántica se deriva de las diferentes combinaciones.

La revisión de la propuesta de Talmy (2000) a la luz de estos modelos permite extender las nociones de Figura y Fondo a diferentes tipos de predicados (ver AcedoMatellán \& Mateu, 2013) en los que el Fondo corresponde a un estado o locación, mientras que la Figura es el argumento del cual se predica ese estado/locación. Ambas interpretaciones surgen de una configuración en la que el constituyente que se ensambla como complemento de Place se interpreta como el Fondo, mientras que el que se ensambla en la posición de especificador se interpreta como Figura. Place es simplemente una denominación para las relaciones predicativas de estado/locación. Cuando la especificación sobre la manera en la que se da esa relación es pertinente, tiene lugar la adjunción de una raíz a una categoría funcional, operación conocida como Conflación [Manner Conflation] (Mateu, 2012). Para el caso de 'ser hermano(s)', por ejemplo, el estado de 'hermandad' se predica con respecto a una Figura. Así, si asumimos que $\sqrt{ } 128$ es la raíz que se materializará como /er.man/, tendríamos las siguientes estructuras argumentales para los objetos sintácticos que encontramos en una oración como 'Los chicos son hermanos' (16) y en 'Esteban es hermano de Miguel' $(17)^{4}$.

(16) Los chicos son hermanos

(17) Esteban es hermano de Miguel
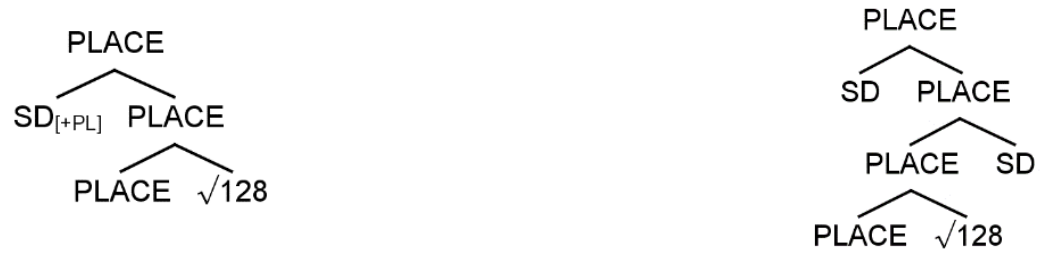

En este punto, surge la discusión con respecto a la interpretación simétrica en relación a los argumentos. Es decir, la interpretación simétrica 'requiere' de la presencia de algún tipo de pluralidad (continua como en (16) o discontinua como en (17)), pero la pluralidad no desencadena una interpretación simétrica o recíproca ('Los chicos se aman' puede interpretarse de manera recíproca o reflexiva). Esta observación es central en los modelos neo-construccionistas en un abordaje como la MD: si la interpretación de las raíces es el resultado del contexto sintáctico en el que aparecen, nada impide que en (16) el SD sea singular; mientras que si la raíz presenta alguna especificación con respecto a la simetría estaríamos ante un modelo que no deja de tener un carácter proyeccionista. 
En la literatura, ha habido desarrollos para las dos opciones. Borer (2003), por ejemplo, considera que todas las combinaciones son gramaticales en el sentido técnico y, en cualquier caso, serán semánticamente anómalas. Embick (2015), siguiendo las discusiones en Embick y Marantz (2008), plantea la posibilidad de que las restricciones en la distribución de las raíces surjan de propiedades semánticas inherentes que estas puedan tener. De esta forma, las anomalías semánticas se producirían cuando esas propiedades no pueden reconciliarse con las contribuciones del contexto sintáctico en el que aparecen las raíces. La diferencia entre estas dos perspectivas es que la primera atribuye propiedades del significado a aspectos conceptuales o pragmáticos, mientras que la segunda restringe estos aspectos a la gramática. En el caso que nos compete, sería un rasgo interpretable como simétrico en relación a $\sqrt{128}$, por ejemplo, el que definiría que 'Los chicos son hermanos' y 'Esteban es hermano de Miguel' tengan interpretación simétrica, mientras que 'Esteban es hermano' sería semánticamente anómala o se salvaría, en algunos casos, recuperando pragmáticamente uno de los argumentos (Juan es amigo/colega/vecino' del hablante/hablante + grupo/comunidad o un antecedente discursivo).

Aquí seguimos una hipótesis más cercana a la de Borer (2003), aunque consideramos que las raíces no son individuables ni semántica ni fonológicamente, sino que son individuables a partir de la computación morfosintáctica (Acquaviva, 2008), por esta razón, a lo largo de la exposición hemos utilizado índices para identificarlas. Así, $\sqrt{128}$ es miembro del conjunto de primitivos sintácticos con los que opera la Sintaxis (Lista 1). En la Lista 2, es decir, el Vocabulario, se encuentran las instrucciones para la pronunciación de los nodos terminales en contexto: $\sqrt{128} \leftrightarrow$ /er.man-/. Finalmente, en la Lista 3, la Enciclopedia, se hallan las instrucciones para la interpretación de esos nodos terminales en un contexto morfosintáctico dado: $\sqrt{128}$ $\leftrightarrow$ 'herman(o)'. El hecho de que existan entidades identificables como raíces, que carecen de significado fuera de su contexto morfosintáctico (-ceive en receive 'recibir', perceive 'percibir o -pose en suppose 'suponer', compose 'componer', etc., en inglés) constituye un fuerte argumento a favor de esta hipótesis ${ }^{5}$. En este sentido, el hecho de que cualquier $\mathrm{SD}$ que se ensamble en relación a $\sqrt{128}$ establezca una relación de simetría, no es el resultado de un rasgo interpretable en $\sqrt{128}$, sino de las 'instrucciones' en la Enciclopedia, que están determinadas también por aspectos conceptuales o pragmáticos (además de los configuracionales).

Finalmente, nos interesa detenernos en la distribución de 'hermano' en distintos contextos morfosintácticos que permiten obtener formas como 'Esteban es hermano de Miguel'/'Esteban es el hermano de Miguel'. La raíz $\sqrt{12} 8_{\text {HERMAN- en estructuras }}$ como (16) y (17) está sujeta a nuevos ensambles con diferentes proyecciones funcionales. En este punto, cabe destacar que los nombres relacionales, como los nombres que denotan capacidades, aparecen de manera productiva en posiciones predicativas como nombres desnudos (Esteban y Miguel son 
hermanos/colegas/amigos/primos'; 'Esteban es hermano/colega/amigo/primo de Miguel'), como ha sido ampliamente señalado en la literatura (Bosque, 1996; Fernández Leborans, 1999; Beyssade \& Dobrovie-Sorin, 2005; Espinal \& Mateu, 2011). Dado que incluso en construcciones predicativas estos nombres admiten modificación prenominal ('Esteban es buen colega') y que, además, presentan información de denotación sexual y de número, consideramos que las proyecciones funcionales que se ensamblan en estos casos son el categorizador nominal $n$ y Núm. Es decir, no hay ensamble con un nodo $\mathrm{D}$, que sí estaría presente cuando la interpretación es referencial ('los hermanos') y no predicativa ('hermanos').

(18) los hermanos

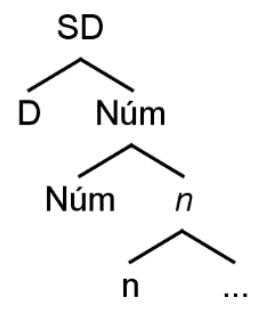

(19) hermanos

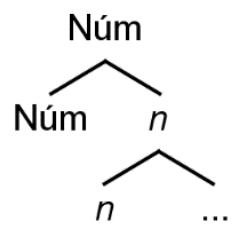

Si bien se ha señalado que los nombres desnudos en construcciones predicativas suelen aparecer especificados (Fernández Leborans, 1999), la cuestión de la especificación parece ser más compleja y estar relacionada con los rasgos de Número (20). Al comparar el uso predicativo con el referencial, surgen además distinciones que vinculan la especificidad con la presencia de la proyección D (21).

(20) a. Esteban y Miguel son hermanos.

b. Esteban es hermano*(de Miguel).

(21) Esteban y Miguel son los hermanos *(de Ana).

La diferencia en los datos de (20) se explica en el modelo que seguimos aquí, como una anomalía en el componente interpretativo. Las raíces que se interpretan en el componente interpretativo como nombres de relación (no) simétrica requieren de, al menos, dos argumentos. Así, (20b) queda descartado en el componente interpretativo, ya que $\sqrt{128}$ en el contexto pertinente está sujeta a la siguiente interpretación: $R(x, y)$, entonces $R(y, x)$, donde $R$ es $\sqrt{ } 128$.

La comparación entre (20a) y (21) llama la atención con respecto a la presencia de determinantes. El artículo definido parece obligar la especificación del nombre, a pesar de tener un argumento que denota una pluralidad ('Esteban y Miguel'). El plural desnudo, en cambio, puede aparecer especificado ('son hermanos de Ana'), sin embargo, ante la ausencia de esa especificación, la interpretación necesaria es la recíproca, a diferencia de lo que sucede con otros nombres relacionales (Juan y 
Esteban son padres' $\neq$ 'Miguel es padre de Esteban' y 'Esteban es padre de Miguel'). En este sentido, el conjunto de datos de (22), resulta pertinente.

(22) a. Esteban y Miguel son hermanos de Ana.

b. Esteban y Miguel son hermanos.

c. Esteban y Miguel son los hermanos de Ana.

d. *Esteban y Miguel son los hermanos.

La presencia del determinante dificulta la lectura recíproca, incluso con un nombre como 'hermano' del que se desprende que si Esteban y Miguel son hermanos de Ana, también lo son entre ellos ${ }^{6}$. Este contraste entre (22a-c) y (22d) parece indicar diferencias sustanciales en cuanto a las proyecciones involucradas y a los objetos sintácticos enviados al componente interpretativo. Es decir, los rasgos vinculados a la proyección D inciden en la interpretación del argumento plural, produciendo en determinados contextos anomalías semánticas. El SD ‘los hermanos' -y también 'el hermano'- puede aparecer en diferentes contextos sin mayores especificaciones y son las relaciones endofóricas las que permiten recuperar las entidades vinculadas en esa predicación. Por lo tanto, proponemos que el SD 'los hermanos' en (22d) presentaría una estructura más simple, en la que no habría una proyección relacional como PLACE. Cuando [ $n$ [ $\sqrt{ } 128]]$ llega al componente interpretativo, las entidades que participan en la relación ' $R(x, y)$, entonces $R(y, x)$ ' se recuperan del contexto y si no se pueden recuperar, el resultado es semánticamente anómalo. En (22c), en cambio, el SD 'los hermanos de Ana' presentaría una estructura sintáctica en la que la proyección PLACE estaría presente. A continuación se muestran las configuraciones correspondientes ${ }^{7}$.

(23) los hermanos

(24) los hermanos de Ana
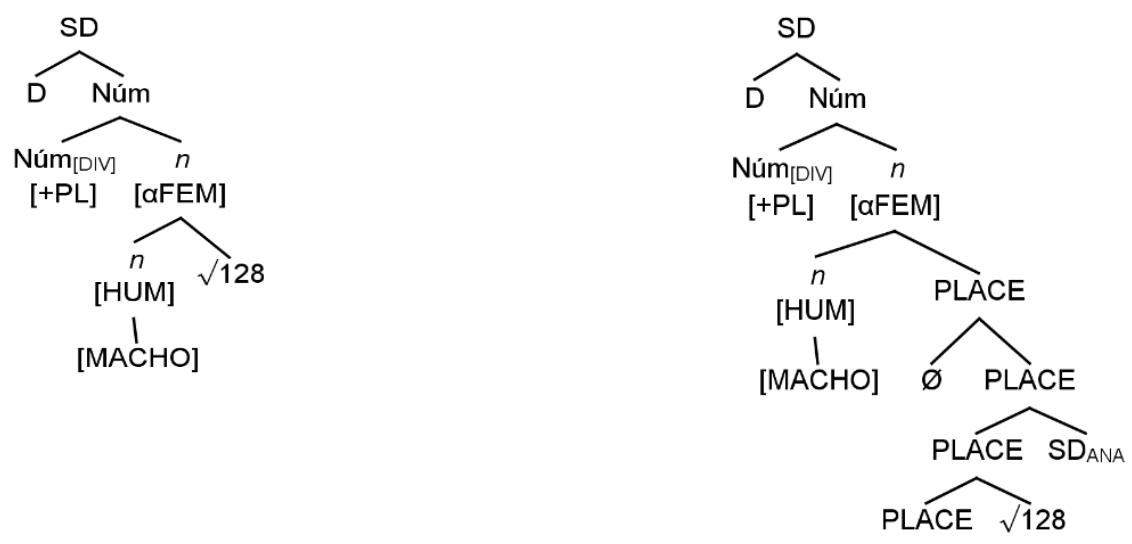


\subsection{Recapitulación}

En este apartado procuramos avanzar en una propuesta con respecto a la estructura de los nombres de relación (no) simétrica, que permita dar cuenta de las alternancias en la realización de los argumentos involucrados. Específicamente, consideramos que PLACE establece una relación de predicación entre (a) dos SSDD o (b) entre un $\mathrm{SD}$ y una raíz. En el primer caso, la raíz se adjunta al núcleo PLACE ('Conflación') y se interpreta como la manera en la que se establece esa relación. En el segundo caso, parece haber un requerimiento de pluralidad con respecto al SD ensamblado en el especificador de PLACE. Sin embargo, consideramos que este no es un requerimiento del componente sintáctico, sino del componente interpretativo. Es decir, la Sintaxis puede formar una estructura de este tipo con un SD en singular, pero al enviar ese objeto sintáctico al componente interpretativo, la configuración resulta anómala, a menos que pueda recuperarse el otro argumento de un contexto discursivo particular.

\section{CONCLUSIONES}

En este artículo, exploramos un análisis para los nombres de relación (no) simétrica que nos permita dar cuenta de la interpretación simétrica, por un lado, y de las alternancias en la estructura argumental, por el otro. El primer aspecto conduce directamente a la discusión sobre el género y el número en español. Con respecto al primero, procuramos mostrar que las tres dimensiones que plantea (Harris, 1991) son fundamentales para entender los dominios de la relación simétrica, de otros aspectos que tienen que ver con la buena formación de una lengua como el español (a nivel morfo-sintáctico y a nivel morfo-fonológico).

En cuanto al número y la estructura argumental de estos nombres, exploramos un análisis neo-construccionista dentro de la Morfología Distribuida, en el que las raíces no están individuadas ni semántica ni fonológicamente. Planteamos, entonces, que el hecho de que no haya especificaciones particulares en las raíces, explicaría las combinaciones posibles, la manera de interpretar los SSDD con denotación plural en construcciones predicativas y la ausencia de argumentos, cuando la estructura presenta una proyección relacional como PLACE. Consideramos que aún quedan muchos otros aspectos por explorar en relación a este tipo de nombres, pero esperamos que estos aportes permitan avanzar con la investigación.

\section{REFERENCIAS BIBLIOGRÁFICAS}

Acquaviva, P. (2008). Roots and lexicality in Distributed Morphology. Ms.: University College Dublin/Universit at Konstanz. [en línea]. Disponible en: http://ling.auf.net/lingBuzz/000654. 
Acedo-Matellán, V. (2016). The morphosyntax of transitions. A case study in Latin and other languages. Oxford: Oxford University Press.

Acedo-Matellán, V. \& Mateu, J. (2013). Satellite-framed Latin vs. verb-framed Romance: A syntactic approach. Probus, 25, 1-39.

Arad, M. (2003). Locality contraints on the interpretation of roots: The case of Hebrew denominal verbs. Natural Language and Linguistic Theory, 21, 737-778.

Beyssade, C. \& Dobrovie-Sorin, C. (2005). A syntax-based analyis of predication. En E. Georgala \& J. Howell (Eds.), Proceedings of SALT XV (pp. 44-61). Ithaca: Cornell University Press.

Bobaljik, J. (2008). Where's $\Phi$ ?: Agreement as a post-syntactic operation. En M. Van Koppen, P. Hendriks, F. Landsbergen, M. Poss \& J. van der Wal (Eds.), Special Issue of Leiden Papers in Linguistics, 3(2), 1-23.

Borer, H. (2003). Exo-skeletal vs. endo-skeletal explanations: Syntactic projections and the lexicon. En J. Moore \& M. Polinsky (Eds.), The nature of explanation in linguistic theory (pp. 31-67). Stanford: CSLI Publications.

Borer, H. (2005). In name only. Oxford: Oxford University Press.

Borer, H. (2009). Roots and categories. Ponencia presentada en el 19 ${ }^{\text {th }}$ Colloquium on Generative Grammar, University of the Basque Country, Vitoria-Gasteiz.

Bosque, I. (1996). Por qué determinados sustantivos no son sustantivos determinados. Repaso y balance. En I. Bosque (Ed.), El sustantivo sin determinación. La ausencia de determinante en la lengua española (pp. 13-120). Madrid: Visor.

Chomsky, N. (1970). Remarks on nominalizations. En R. Jacobs \& P. Rosenbaum (Eds.), Readings in English transformational grammar (pp. 184-221). Waltham Massachusetts: Ging y Co.

Chomsky, N. (2001). Derivation by Phase. En M. Kenstowicz (Ed.), Ken Hale. A Life in Language (pp. 1-52). Cambridge, Massachusetts: MIT Press.

Chomsky, N. (2008). On phases. En R. Freidin, P. Otero \& M. L. Zubizarreta (Eds.), Foundational Issues in Linguistic Theory. Essays in Honor of Jean-Roger Vergnaud (pp. 133-166). Cambridge, MA: MIT Press.

Demonte, V. (2015). Parámetros y variación en la interfaz léxico-sintaxis. En Á. Gallego (Ed.), Perspectivas de sintaxis formal (pp. 391-430). Madrid: AKAL.

Embick, D. (2015). The morpheme. A theoretical introduction. Berlín: De Gruyter Mouton.

Embick, D. \& Marantz, A. (2008). Architecture and blocking. Linguistic Inquiry, 39(1), $1-53$. 
Embick, D. \& Noyer, R. (2005). Distributed Morphology and the Syntax/Morphology Interface. Ms. University of Pennsylvania.

Espinal, T. \& Mateu, J. (2011). Bare nominals and argument structure in Catalan and Spanish. The Linguistic Review, 28, 1-39.

Estomba, D. (2017). El género sintáctico y la proyección del nombre en español. Neuquén: EDUCO, Serie Tesis de Maestría.

Fernández Leborans, M. J. (1999). La predicación: Las oraciones copulativas. En I. Bosque \& V. Demonte (Dirs.), Gramática descriptiva de la lengua española (pp. 2357-2460). Madrid: Espasa.

Hale, K. \& Keyser, S. J. (1993). On argument structure and the lexical expression of syntactic relations. En K. Hale \& S. J. Keyser (Eds.), The View from Building 20: Essays in Linguistics in Honor of Sylvain Bromberger (pp. 53-109). Cambridge, Massachusetts: MIT Press.

Halle, M. (1997). Distributed Morphology: Impoverishment and fission. MIT Working Papers in Linguistics, 30, 425-449.

Halle, M. \& Marantz, A. (1993). Distributed Morphology and the pieces of inflection. En K. Hale \& S. Keyser (Eds.), The View from Building 20 (pp. 111-176). Cambridge, Mass.: MIT Press.

Harley, H. \& Noyer, R. (2000). Licensing in the non-lexicalist lexicon. En B. Peeters (Ed.), The Lexicon/Encyclopaedia Interface (pp. 349-374). Ámsterdam: Elsevier.

Harris, J. (1991). The exponence of gender in Spanish. Linguistic Inquiry, 22, 65-88.

Harris, J. (1992) The form classes of Spanish sustantives. En G. Booij \& J. van Marle (Eds.), Yearbook of Morphology, 1991 (pp. 65-88). Netherlands: Kluwer Academic Publishers.

Kihm, A. (2005). Noun class, gender, and the lexicon-syntax-morphology interfaces: A comparative study of Niger-Congo and Romance languages. En G. Cinque \& R. Kayne (Eds.), Comparative Syntax (pp. 459-512). Oxford: Oxford University Press.

Marantz, A. (1997). No escape from syntax: Don't try morphological analysis in the privacy of your own lexicon. En A. Dimitriadis, L. Siegel, C. Surek-Clark \& A. Williams (Eds.), Proceedings of the 21st Penn Linguistics Colloquium (pp. 201-225). Philadelphia: UPenn Working Papers in Linguistics.

Marantz, A. (2001). Words. Cambridge, Massachusetts: Massachusetts Institute of Technology. 
Marantz, A. (2007). Phases and words. En S. H. Choe (Ed.), Phases in the theory of grammar (pp. 191-222). Seúl: Dong-In Publishing Co.

Marantz, A. (2013). Verbal argument structure: Events and participants. Lingua, 130, 152-168.

Mateu, J. (2002). Argument structure. Relational construal at the syntax-semantics interface. Tesis doctoral, Universitat Autònoma de Barcelona, Barcelona, España.

Mateu, J. (2012). Conflation and incorporation processes in resultative constructions. En V. Demonte \& L. McNally (Eds.), Telicity, Change, and State. A CrossCategorial View of Event Structure (pp. 252- 278). Oxford/Nueva York: Oxford University Press.

Partee, B. H. (2008). Symmetry and symmetrical predicates. En A. E. Kibrik, V. I. Belikov \& B. V. Dobrovolsky (Eds.), Computational linguistics and intellectual technologies: Papers from the international conference "Dialogue" (pp. 606-611). Moscow: Institut Problem Informatiki.

Percus, O. (2011). Gender features and interpretation: A case study. Morphology, 21, 167-196.

Saab, A. (2004). El dominio de la elipsis nominal en español: Identidad estricta e inserción tardía. Tesis de magíster, Universidad Nacional del Comahue, Neuquén, Argentina.

Saab, A. (2008). Hacia una teoría de la identidad parcial en la elipsis. Tesis doctoral, Universidad de Buenos Aires, Buenos Aires, Argentina.

Siddiqi, D. (2009). Syntax within the word. Ámsterdam/Philadelphia: John Benjamins.

Staroverov, P. (2007). Relational nouns and reciprocal plurality. En T. Friedman \& M. Gibson (Eds.), Actas SALT 17 (pp. 300-316). Ithaca, Nueva York: Cornell University.

Talmy, L. (2000). Toward a cognitive semantics, vol. 2: Typology and Process in Concept Structuring. Cambridge, Massachusetts: MIT Press.

\section{NOTAS}

${ }^{1}$ En principio, seguimos el sistema de rasgos binarios [+/- fem] (Saab, 2004; Estomba, 2017, entre muchos otros) y no el de rasgos privativos de Harris (1991). A lo largo de la presentación explicitamos algunas especificaciones con respecto a la marcación del rasgo [fem]. 
${ }^{2}$ Cabe destacar que parte de la composicionalidad que da lugar a la lectura genérica en (12a) es la selección de un argumento nulo. La sola presencia de una expresión referencial plena como argumento del nombre relacional anula la interpretación genérica $y$, al mismo tiempo, la denotación sexual amplia del masculino singular.

3 Agradecemos a uno de los evaluadores anónimos quien nos señala que una gramática que distingue tipos de nominalizadores, como la que proponemos, recibe evidencia empírica en contrastes como man/es (alemán) y on/il (francés), ya que los primeros miembros de cada par (man y on) son formas pronominales que tienen referencia únicamente humana, mientras que los segundos (es e i $)$ podrían presentar estructuras con un nominalizador no especificado. Asimismo, nos sugiere vincular esta propiedad con la conversión categorial de adjetivos a sustantivos que resulta más sistemática cuando la lectura es humana, lo cual podría deberse a restricciones con respecto a la legitimación de la estructura, en la que la presencia de un nominalizador [HUM] sería indispensable.

${ }^{4}$ Utilizamos PLACE en el sentido desarrollado por Acedo-Matellán (2016), es decir, como una capa relacional que introduce una predicación, contra la presencia de dos capas relacionales (PATH y PLACE) que implicarían una transición (cambio de estado/locación). Esto significa que no estamos asumiendo una semántica locativa para esta proyección.

${ }^{5}$ En español encontramos formas como 'poner', que claramente se vinculan con una raíz, pero que también su interpretación depende del contexto morfosintáctico: 'deponer', 'componer', 'posponer', 'suponer'. Evidentemente, si la raíz correspondiente a 'poner' estuviera especificada con algún tipo de rasgo, deberíamos considerar que las formas 'deponer', 'componer', etc. se forman a partir de primitivos diferentes.

${ }^{6}$ Esta interpretación no se desprende de todos los nombres de relación simétrica. Los nombres como 'hermano', 'colega', 'vecino' la admiten (los dos últimos si se emplean las formas de presente), mientras que 'amigo', 'amante', 'primo' o bien no la desencadenan ('Miguel y Esteban son los amantes de Carmela', no implica que 'Miguel y Esteban sean amantes entre sí') o bien están sujetos a restricciones que exceden una discusión sintáctica.

7 Dejamos una categoría vacía en el lugar de especificador de PLACE para indicar que la relación de predicación 'ser hermano de Ana' se establece también en este caso. Queda abierta para futuras investigaciones la discusión con respecto a la extracción del dominio del SD como una posible solución a al problema de la referencia.

\section{AGRADECIMIENTOS}

* Quiero agradecer especialmente a los evaluadores anónimos por sus enriquecedores comentarios y sugerencias. Va también mi agradecimiento a Walter Koza, editor del número monográfico en el que se inscribe este artículo. Esta investigación ha sido financiada por el Consejo Nacional de Investigaciones Científicas y Técnicas (CONICET). 